\title{
STANDARDIZED SLOW ENTERAL FEEDING PROTOCOL AND INCIDENCE OF NECROTIZING ENTEROCOLITIS IN EXTREMELY LOW BIRTH WEIGHT INFANTS
}

By

SREEKANTH K. VISWANATHAN, MD

Submitted in partial fulfillment of the requirements for the degree of Master of Science

Thesis Advisors

Marc Collin, MD

Sharon Groh-Wargo, PhD, RD, LD

Dennis Super, MD,MPH

Douglas Einstadter, MD,MPH

\section{Clinical Research Scholars Program CASE WESTERN RESERVE UNIVERSITY \\ August 2014}




\title{
CASE WESTERN RESERVE UNIVERSITY \\ SCHOOL OF GRADUATE STUDIES
}

\author{
We hereby approve the thesis/dissertation of \\ SREEKANTH K. VISWANATHAN, M.D. \\ candidate for the degree of Master of Science * \\ Committee Chair \\ Marc Collin, MD. \\ Committee Member \\ Sharon Groh-Wargo, PhD, RD, LD. \\ Committee Member \\ Dennis Super, MD, MPH.
}

Committee Member

Douglas Einstadter, MD, MPH.

Date of Defense

June 17, 2014

*We also certify that written approval has been obtained for any proprietary material contained therein. 


\section{TABLE OF CONTENTS}

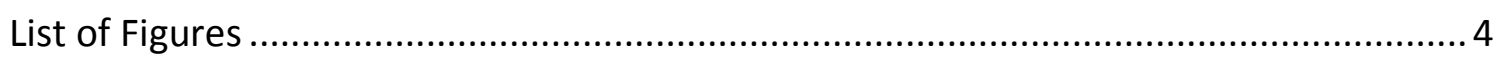

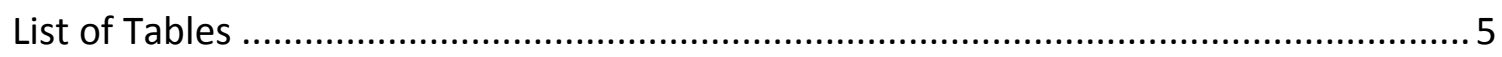

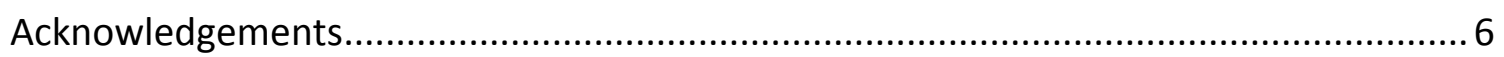

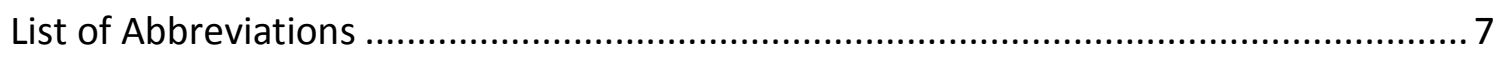

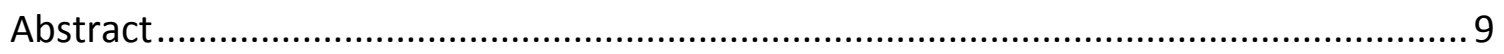

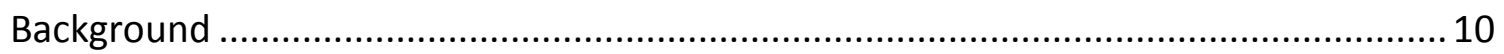

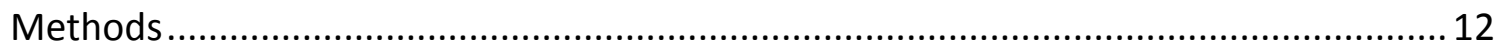

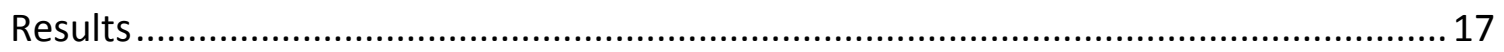

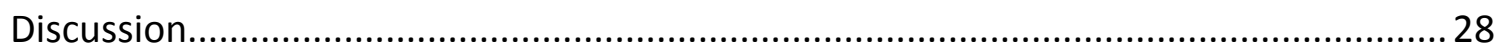

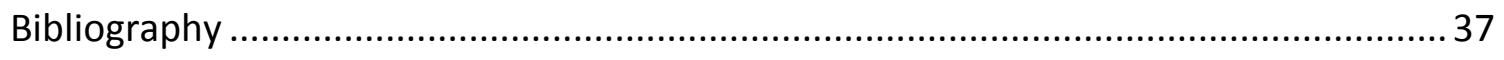




\section{LIST OF FIGURES}

FIGURE 1: Incidence of NEC,Surgical NEC and NEC/Death in infants with birth weight

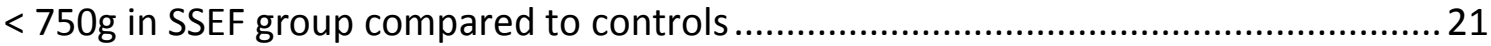




\section{LIST OF TABLES}

Table 1:

Enteral feeding practices for ELBW infants during the respective study ....................... 14

Table 2:

Demographics of Control and SSEF group ELBW infants ...................................... 18

Table 3:

Nutritional characteristics of Control and SSEF group ELBW infants ..........................19

Table 4:

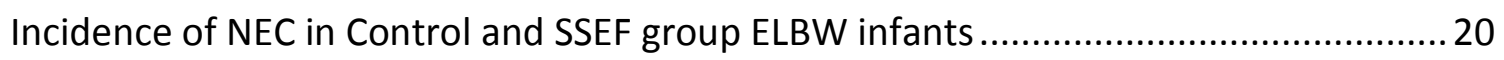

Table 5:

Co-morbidities observed in Control and SSEF group ELBW infants ............................22

Table 6:

NICU discharge outcomes of Control and SSEF group ELBW infants ...........................24

Table 7:

Propensity score adjusted outcomes in SSEF group compared to controls ....................26 


\section{ACKNOWLEDGEMENTS}

I would like to express my sincere gratitude to the following for their support and encouragement throughout the duration of this project and the Master's program:

- Dr. Marc Collin: Mentor and Thesis Committee Chair

- Drs. Sharon Groh-Wargo and Dennis Super: Thesis Committee members

- Dr. Douglas Einstadter: CRSP Advisor and Thesis Committee member

- All of my patients and their families who motivate me every day to strive for clinical and research excellence. 


\section{LIST OF ABBREVIATIONS}

NEC: Necrotizing enterocolitis

SSEF: Slow standardized enteral feeding

VLBW: Very low birth weight $(<1500 \mathrm{~g})$

ELBW: Extremely low birth weight $(<1000 \mathrm{~g})$

NICHD: National Institute of Child Health and Development

NICU: Neonatal intensive care unit

MHMC: MetroHealth Medical Center

GA: Gestational age

BW: Birth weight

SGA: Small for gestational age

SNAP-PE: Score of Neonatal Acute Physiology - Perinatal Extension

UAC: Umbilical arterial catheter

UVC: Umbilical venous catheter

PICC: Peripherally inserted central catheter

PN: Parenteral nutrition

MM: Mother's milk

DBM: Donor breast milk

NPO: Nil per os

PS: Propensity score

RCT: Randomized controlled trial

CLD: Chronic lung disease 
PDA: Patent ductus arteriosus

CONS: Coagulase negative staphylococcal sepsis

CSF: Cerebrospinal fluid

CLABSI: Central line-associated bloodstream infections

ALP: Alkaline phosphatase

IVH: Intra-ventricular hemorrhage

ROP: Retinopathy of prematurity 


\title{
Standardized Slow Enteral Feeding Protocol and Incidence of Necrotizing Enterocolitis in Extremely Low Birth Weight Infants.
}

\section{By}

SREEKANTH K. VISWANATHAN, MD

\begin{abstract}
To study the effects of a standardized slow enteral feeding (SSEF) on the incidence of necrotizing enterocoltis (NEC), we compared outcomes for extremely low birth weight infants who followed a SSEF protocol $(n=125)$ to a similar group of historical controls $(n=294)$. There was no significant difference in the rate of NEC (5.6 vs. $11.2 \%$, $p=0.10$ ) between the SSEF and historical groups. However, the rate of NEC in infants with birth weight $<750$ g was significantly less in the SSEF group compared to controls (2.1 vs. $16.2 \%, p<0.01$ ). The combined NEC/death was significantly less in the SSEF group (10.4 vs.19.4\%, p=0.03). However, in subgroup analysis, this effect was present only in infants with birth weight $<750 \mathrm{~g}(12.8 \%$ vs. $29.5 \%, \mathrm{p}=0.03)$. Compared to controls, feeding initiation day, days to full enteral feeding, parenteral nutrition days and the total central line days were all longer in the SSEF group. Compared to a historical feeding protocol, the use of a SSEF protocol was associated with significantly reduced incidence of NEC and combined NEC/death in infants with birth weight $<750 \mathrm{~g}$.
\end{abstract}




\section{BACKGROUND}

Necrotizing enterocolitis (NEC) continues to be a devastating neonatal illness especially in extremely low birth weight (ELBW, birth weight $\leq 1000 \mathrm{~g}$ ) preterm infants. $^{1,2,3}$ In very low birth weight (VLBW, birth weight $<1500 \mathrm{~g}$ ) infants, the mean incidence of NEC ranges from 7 to $9 \%$ with an estimated case fatality rate of $15-30 \%$ with the greatest mortality in infants requiring surgery for NEC. ${ }^{1,4,5,6,7}$ In the most recently published NICHD research network outcome data, the incidence of NEC was $11 \%$ for preterm infants born at less than 28 weeks gestation. ${ }^{8}$ In addition to short-term complications such as feeding intolerance, intestinal obstruction, and short-gut syndrome, surviving infants, particularly infants with surgical NEC, have poorer neurodevelopmental outcomes, and represent a huge financial burden to the health care system. $^{9}$ The pathophysiology of NEC is poorly understood, but it is likely a multifactorial disease. ${ }^{7,10}$ Immaturity of the intestinal tract, inappropriate responses to injury, abnormal bacterial colonization and genetic predisposition have all been implicated in the etiology of NEC. ${ }^{7}$ Since little progress has been made in the management of NEC once it occurs, preventive strategies are more likely to have a greater impact in reducing the mortality and morbidity from NEC. ${ }^{11}$

Most preterm infants who develop NEC have received enteral feeds. However, it remains unclear which aspects of feeding regimens affect the risk of NEC. Significant practice variation exists as to when feeds are initiated, how they are advanced, and how feeding intolerance is managed in preterm infants. ${ }^{2}$ The modifiable risk factors related 
to enteral feeding for the development of NEC in preterm infants include the timing of introduction of feeds, the duration of trophic feeding and the rate of advancement of feeding and the type of milk human milk versus formula feeding. ${ }^{12,13}$ Standardizing the feeding regimen itself has been shown to reduce the incidence of NEC. ${ }^{13}$

An immature enteric nervous system and intestinal dysmotility warrant gradual and cautious increments in enteral feedings. Observational studies have reported a higher incidence of NEC in centers where enteral feeding is introduced earlier and feeding volumes are advanced more quickly. ${ }^{12,13,14,15}$ Pietz et al reported a $0.4 \%$ incidence of NEC in 1158 VLBW infants ( 60\% were ELBW infants) who followed a late-onset, slow, continuous drip feeding protocol. ${ }^{15}$ In contrast, a recent meta-analysis of 5 randomized controlled trials comparing slow advancement $(<15-20 \mathrm{ml} / \mathrm{kg} /$ day $)$ versus faster advancement (30-35 ml/kg/day) did not detect any significant difference in NEC or allcause mortality. ${ }^{16}$ However, ELBW infants were only included in two of the studies and both had broad exclusion criteria ${ }^{17,18}$ Due to the limited number of ELBW infants enrolled in these studies, caution must be used when generalizing these results to all ELBW infants, the group at highest risk for developing NEC. ${ }^{16}$

The precise effect of enteral feeding advancement on occurrence of NEC has not yet been thoroughly investigated in ELBW infants. We hypothesized that exposure to a standardized slow enteral feeding (SSEF) protocol might reduce the incidence of NEC in ELBW infants without inducing significant adverse events. To investigate this hypothesis we carried out a prospective study in a cohort of ELBW infants who followed the SSEF 
protocol and compared the short-term outcomes with a historical control group of ELBW infants admitted to the same NICU prior to implementation of the SSEF protocol.

\section{METHODS}

The study took place in the Level III NICU at the MetroHealth Medical Center (MHMC). The NICU receives nearly 600 admissions per year including approximately 50 ELBW infants and serves a diverse underserved inner city population in Cleveland, Ohio. The non-standardized feeding guideline for ELBW infants admitted to the MHMC NICU was replaced by the SSEF protocol in August, 2009 (see Table 1 for protocol description). We stratified ELBW infants into 2 weight groups (< $750 \mathrm{~g}$ birth weight and $750-1000 \mathrm{~g}$ birth weight) and separate SSEF protocols were developed for each group. The SSEF protocols differed from previous feeding guidelines by delaying the start of enteral feeding, with more days to prime the intestine and more cautious increments in feeding. In addition, powdered human milk fortifier (HMF) was introduced earlier during the SSEF protocol when the enteral feed reached $100 \mathrm{ml} / \mathrm{kg} /$ day compared to 150 $\mathrm{ml} / \mathrm{kg} /$ day with the previous guideline. At the attending physician's discretion, initiation of feeds was allowed to be delayed, if indicated, but no infant, was fed sooner than the designated times specified by the protocol. Feeding intolerance was defined as gastric residuals more than $2 \mathrm{ml}$ for infants $<750 \mathrm{~g}$ birth weight and $3 \mathrm{ml}$ for infants between 750-1000 g birth weight, or greater than $50 \%$ of the prior feeding, bile or blood-stained aspirates, abdominal distention/tenderness or presence of blood in the stool. Feeding intolerance was quantified by the number of days feeding were withheld $\geq 24$ hours. 
The incidence of NEC in ELBW infants of $\leq 30$ weeks of birth gestation admitted to the MHMC NICU between September, 2009 and December, 2012 who followed the SSEF protocol (SSEF group, prospective cohort), was compared to ELBW infants of $\leq 30$ weeks of gestation admitted to the MHMC NICU between January, 2003 and July 2009, who followed a non-standardized feeding guideline (historical controls). We excluded infants with major anomalies/known gastrointestinal anomalies, operative diagnosis of spontaneous intestinal perforation, as well as infants who developed NEC/expired before initiation of feeds or were transferred to another facility before reaching full enteral feeding. PN with minimum $1 \mathrm{~g} / \mathrm{kg} /$ day protein was initiated on admission to the NICU during the whole study period. Protein content of starter PN was increased to $2.5 \mathrm{~g} / \mathrm{kg} /$ day half-way through the SSEF study period. Intravenous fat emulsion was discontinued when enteral feedings reached $100 \mathrm{ml} / \mathrm{kg} /$ day and $\mathrm{PN}$ was discontinued when enteral feedings reached $120 \mathrm{ml} / \mathrm{kg} /$ day. Human milk feeding was encouraged and, if not available, standard preterm formula (24 cal/oz) was used. Donor breast milk or probiotic preparations were not used during the study period.

The incidence of NEC in ELBW infants of $\leq 30$ weeks of birth gestation admitted to the MHMC NICU between September, 2009 and December, 2012 who followed the SSEF protocol (SSEF group, prospective cohort), was compared to ELBW infants of $\leq 30$ weeks of gestation admitted to the MHMC NICU between January, 2003 and July 2009, who followed a non-standardized feeding guideline (historical controls). We excluded infants with major anomalies/known gastrointestinal anomalies, operative diagnosis of spontaneous intestinal perforation, as well as infants who developed NEC/expired 
before initiation of feeds or were transferred to another facility before reaching full enteral feeding

Table 1: Enteral feeding practices for ELBW infants during the respective study period

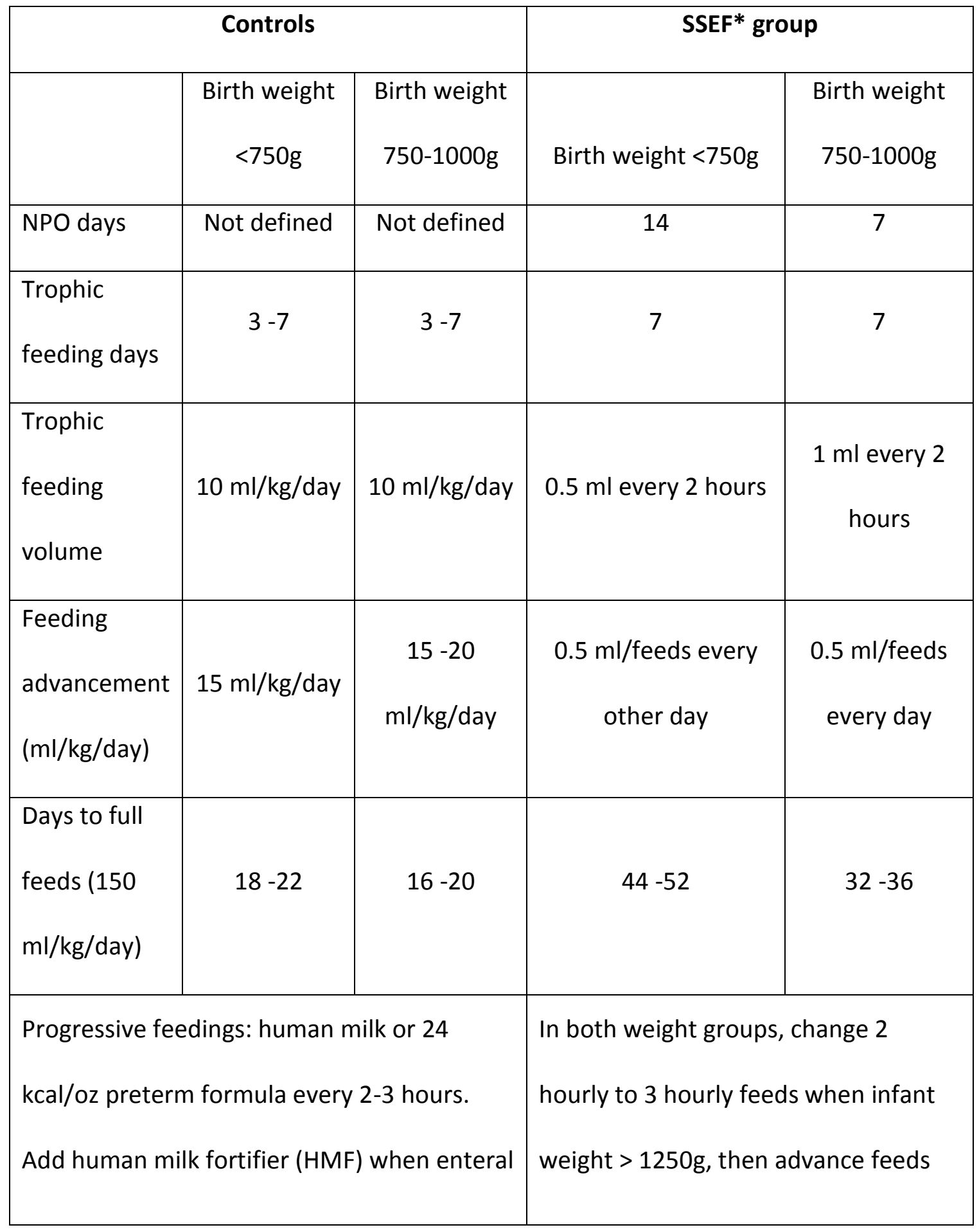




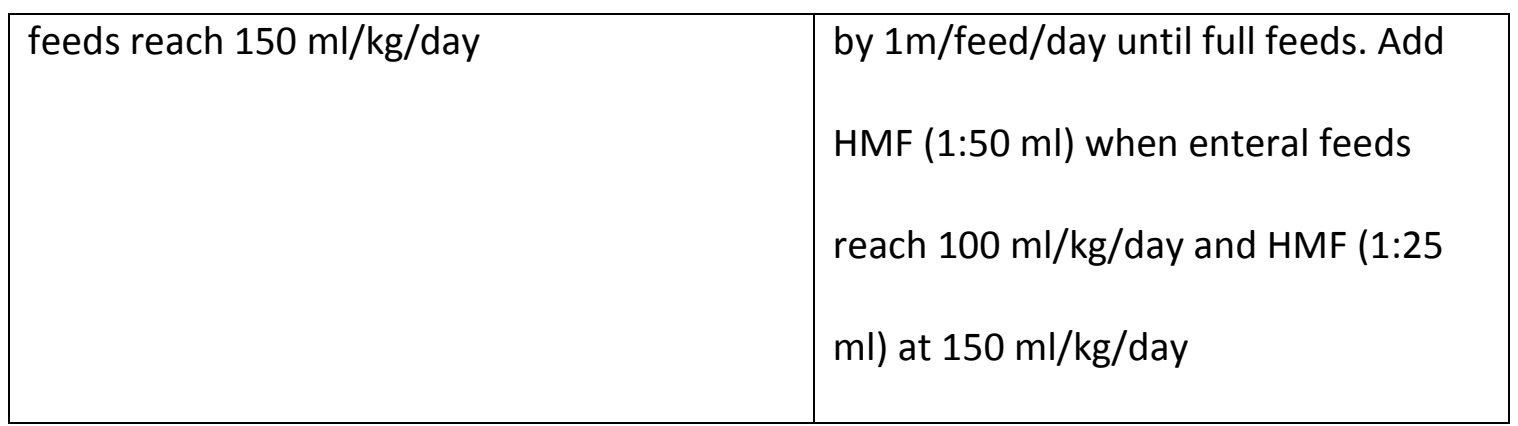

*SSEF-Slow standardized enteral feeding

The primary outcome of the study was the incidence of NEC in ELBW infants. We defined the occurrence of NEC as Bell Stage 2 or greater as per the modified Bell staging $^{19}$ or diagnosed at surgery. Secondary outcomes included the incidence of NEC or death combined, discharge weight, late-onset sepsis, cholestasis and metabolic bone disease of prematurity. We reviewed all medical records for infants' demographics [including gestational age (GA), birth weight (BW), small for gestational age (SGA), gender, race, mode of delivery, Apgar scores at 1 and 5 minutes, exposure to antenatal steroids and chorio-amnionitis and severity of illness measured by the Score of Neonatal Acute Physiology - Perinatal Extension (SNAP-PE)]. The SNAP-PE score is a 9-item neonatal illness severity and mortality risk score. ${ }^{20}$ It is calculated from data collected on the day of admission to the NICU with points given for physiological items, birth weight, low Apgar score, and SGA. Nutritional data collected includes enteral feeding initiation day, trophic feeding days, trophic feeding volume, days to reach full enteral feeding (150 ml/kg/day), human milk use, parenteral nutrition (PN) days and the duration of central line use [umbilical arterial line (UAC), umbilical venous line (UVC), peripherally inserted central catheter (PICC), Broviac lines, and total central line days (excluding UAC days)]. We also reviewed the medical records for the number of days of mechanical 
ventilation, the incidence of chronic lung disease (CLD, oxygen requirement at 36 weeks of corrected gestation), intra-ventricular hemorrhage (IVH), blood or cerebrospinal fluid (CSF) culture positive sepsis/meningitis, cholestasis (direct bilirubin levels), metabolic bone disease (serum alkaline phosphatase (ALP) levels), patent ductus arteriosus (PDA), retinopathy of prematurity (ROP) and use of medications (postnatal steroids, inotropes, antibiotics, ibuprofen/indomethacin). Late-onset sepsis/meningitis was defined as clinical signs and symptoms consistent with sepsis occurring more than 3 days after birth associated with isolation of a causative organism from at least one blood culture or cerebrospinal fluid. Patients' outcomes including mortality rate, length of hospital stay and weight at discharge from the NICU (among survivors) were recorded. The study was approved by the Institutional Review Board at MetroHealth Medical Center.

\section{STATISTICAL ANALYSIS}

Based on the historic data, the incidence of NEC in ELBW infants was $11 \%$ at the study initiation (MHMC NICU statistics). We determined that a prospective group sample size of 125 ELBW infants compared to 300 controls would have $80 \%$ power to detect a decrease in the incidence of NEC to $3.3 \%$, using a two-tailed $95 \%$ confidence level. This low value for the incidence of NEC was chosen based on the incidence of NEC observed in prior studies using a comparable late-onset slow enteral feeding. ${ }^{15}$ We performed appropriate bivariate analysis to identify the unadjusted differences between the SSEF group and historical controls. All quantitative data are expressed as the mean \pm standard deviation (SD), or median with interquartile range. A $p$ value less 
than 0.05 was considered to be statistically significant. We also performed a propensity score (PS) analysis to calculate the adjusted SSEF group effect compared to controls on various outcomes. PS analysis represents an improvement over traditional modeling strategies. With PS methods, infants in the SSEF group are matched on a range of potentially confounding factors to infants in the control group. The two groups can then be considered equivalent to each other if no statistical difference exists between the groups on all the covariates included in the model. The two PS matched groups are then compared to each other on various outcomes, thus PS analysis represents a quasirandomized control design using observational data. Using a multiple logistic regression including baseline demographic and nutritional variables we calculated a PS for entering the SSEF group for each infant. Analysis using 1:1 greedy PS matching stratified by birth weight group as well as PS weighting by the inverse PS to calculate the average intervention effect were performed. Conditional logistic regression/paired t- test (PS matching) and survey design (PS weighting) was performed based on the PS matched/weighted pairs, as well as direct PS adjusted comparisons of the SSEF group to the controls on NEC and other secondary outcomes. Statistical software ' $R$ ' version $\underline{R}$ 2.14.1 was used for the statistical analysis of the data.

\section{RESULTS}

During the control study period, 391 ELBW infants were admitted to the MHMC NICU.A total of 97 infants met the exclusion criteria (72 infants died and 3 developed NEC before the initiation of enteral feeds, 3 infants were diagnosed with spontaneous 
intestinal perforation, 13 infants were $\geq 31$ week birth gestation, 5 infants were transferred out before reaching full enteral feeds and 1 infant had a major congenital anomaly). During the SSEF study period, 145 ELBW infants were admitted to MHMC NICU and 20 of them met the exclusion criteria (17 infants died before the initiation of enteral feeds, 3 infants were $\geq 31$ week birth gestation). The final study sample consisted of 294 infants in the control group and 125 infants in the SSEF group.

The demographics of the control and SSEF group patients are shown in Table 2. Overall there were no significant differences between the SSEF group and controls except there were more male infants in the SSEF group (Table 2).

Table 2: Demographics of Control and SSEF group ELBW infants

\begin{tabular}{|l|c|c|c|}
\hline & Controls (n=294) & SSEF group (n=125) & p value \\
\hline Birth weight (g) & $754.1 \pm 147.7$ & $766.8 \pm 155.5$ & 0.44 \\
\hline Birth weight percentile & $34.4 \pm 25.1$ & $38.6 \pm 25.6$ & 0.12 \\
\hline Birth gestation (weeks) & $25.9 \pm 1.8$ & $25.7 \pm 1.8$ & 0.33 \\
\hline Gender (Male) & $48.9 \%$ & $60.8 \%$ & 0.03 \\
\hline Ethnicity (African American) & $60.5 \%$ & $57.6 \%$ & 0.59 \\
\hline Small for gestational age & $20.7 \%$ & $25.6 \%$ & 0.30 \\
\hline Apgar 1 minute* & $4(3-6)$ & $4(2-6)$ & 0.05 \\
\hline Apgar 5 minute* & $7(6-8)$ & $7(6-8)$ & 0.10 \\
\hline Caesarian delivery & $67.8 \%$ & $69.6 \%$ & 0.74 \\
\hline
\end{tabular}




\begin{tabular}{|l|c|c|c|}
\hline Antenatal steroids & $75.8 \%$ & $75.2 \%$ & 0.99 \\
\hline Chorioamnionitis & $17.3 \%$ & $14.4 \%$ & 0.56 \\
\hline SNAP-PE Score & $44.3 \pm 14.7$ & $40.9 \pm 16.4$ & 0.05 \\
\hline
\end{tabular}

* Median with interquartile range

Table 3: Nutritional characteristics of Control and SSEF group ELBW infants

\begin{tabular}{|l|c|c|c|}
\hline & $\begin{array}{c}\text { Controls } \\
(\mathbf{n = 2 9 4 )}\end{array}$ & group(n=125) & p value \\
\hline Enteral feeds initiation day & $11.1 \pm 7.1$ & $14.2 \pm 7.3$ & $<0.001$ \\
\hline Trophic feeding days & $5.3 \pm 3.2$ & $7.9 \pm 1.6$ & $<0.01$ \\
\hline NPO days (feeds start to full feeds) & $6.0 \pm 9.4$ & $5.2 \pm 6.6$ & 0.368 \\
\hline Enteral full feed day & $35.1 \pm 19.3$ & $63.6 \pm 19.1$ & $<0.001$ \\
\hline Any human milk use & $68.1 \%$ & $80.2 \%$ & 0.013 \\
\hline Human milk to formula before full & $36.8 \%$ & $53.6 \%$ & 0.001 \\
\hline feeds & & & \\
\hline Human milk on full feeds & $38.3 \%$ & $28.5 \%$ & 0.073 \\
\hline Regain birth weight day & $15.9 \pm 6.3$ & $17.1 \pm 6.5$ & 0.095 \\
\hline Weight on full feed(g) & $1046.5 \pm 345.6$ & $1665.9 \pm 413.1$ & $<0.001$ \\
\hline Parenteral nutrition days & $36.3 \pm 22.5$ & $62.7 \pm 22.9$ & $<0.001$ \\
\hline
\end{tabular}

Compared to controls, the SSEF group had significantly delayed initiation of enteral feeds, took more days to reach full enteral feeds and required more PN days 
(Table 3). The rate of human milk initiation was greater in the SSEF group, but in both groups the majority of infants were on formula when they reached full enteral feeds (Table 3).

The overall incidence of NEC (5.6 vs.11.2 \%) and surgical NEC (1.6 vs. $4.8 \%$ ) were not significantly different between the SSEF group and controls (Table 4). However, in infants with $<750 \mathrm{~g}$ birth weight, there was significant reduction in NEC ( 2.1 vs. $16.2 \%, p$ $<0.01$ ) in the SSEF group compared to controls (Table 4) (Figure 1). No infants $<750 \mathrm{~g}$ birth weight in the SSEF group developed surgical NEC compared to $7.8 \%$ in controls (Figure 1). NEC onset day was significantly delayed in the SSEF group compared to controls [57.9 \pm 23.7 (range 28-97) vs. $31.2 \pm 14.9$ (range 9-66), $p=0.02]$. The incidence of NEC before reaching full enteral feeds was similar between the SSEF group and controls (57.1 vs. $54.5 \%, p>0.99)$.

Table 4: Incidence of NEC in Control and SSEF group ELBW infants

\begin{tabular}{|l|c|c|c|c|}
\hline & Controls & SSEF group & Odd Ratio (CI) & p-value \\
\hline All ELBW infants & & & & \\
Overall NEC $\geq$ stage 2 & $33 / 294(11.2 \%)$ & $7 / 125(5.6 \%)$ & $0.47(0.17-1.12)$ & 0.10 \\
Surgical NEC & $14 / 294(4.8 \%)$ & $2 / 125(1.6 \%)$ & $0.33(0.04-1.45)$ & 0.17 \\
\hline Birth weight $<750 g$ & & & & \\
Overall NEC $\geq$ stage 2 & $21 / 129(16.2 \%)$ & $1 / 47(2.1 \%)$ & $0.11(0.002-0.74)$ & $<0.01$ \\
Surgical NEC & $10 / 129(7.8 \%)$ & $0 / 47(0.0 \%)$ & $0.00(0.000-1.18)$ & 0.06 \\
\hline
\end{tabular}




\begin{tabular}{|l|c|c|c|c|}
\hline Birth weight 750-1000g & & & & \\
Overall NEC $\geq$ stage 2 & $12 / 165(7.3 \%)$ & $6 / 78(7.7 \%)$ & $1.06(0.31-3.20)$ & 0.99 \\
Surgical NEC & $4 / 165(2.4 \%)$ & $2 / 78(2.6 \%)$ & $1.06(0.09-7.57)$ & 0.99 \\
\hline
\end{tabular}

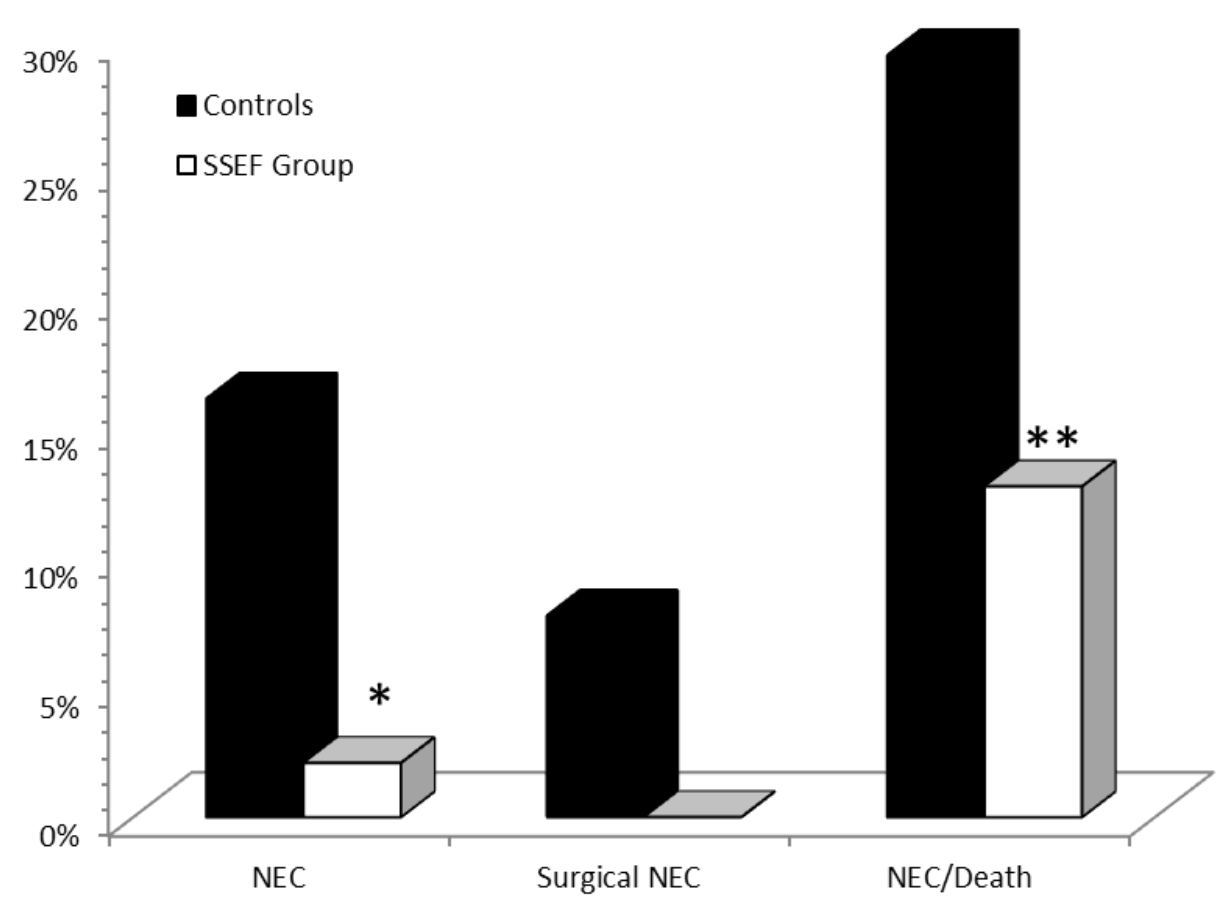

Figure 1: Incidence of NEC, Surgical NEC and NEC/Death in infants with birth weight $<750$ g in SSEF group compared to controls. $* p<0.01, * * p=0.03$

The SSEF group required significantly more total central line days compared to controls $(60.1 \pm 29$ vs. $34.4 \pm 31, p<0.001)$ during their NICU stay (Table 5). The incidence of culture- positive sepsis was not significantly different between the two groups, but infants in the SSEF group developed infection later in their NICU stay and 
were exposed to a longer duration of antibiotics (Table 5). None of the SSEF group infants with NEC had concomitant sepsis compared to $33 \%$ in controls $(p=0.16)$. The SSEF group had significantly higher peak ALP levels with no difference in incidence of cholestasis compared to controls (Table 5). The SSEF group had less CLD, hypotension requiring inotropic medications, PDA ligation and ROP laser surgery compared to the controls (Table 5).

Table 5: Co-morbidities observed in Control and SSEF group ELBW infants

\begin{tabular}{|l|c|c|c|}
\hline & Controls & SSEF group & p value \\
\hline Any sepsis & $44.2 \%$ & $42.4 \%$ & 0.75 \\
Late-onset sepsis & $42.9 \%$ & $40.8 \%$ & 0.75 \\
CONS sepsis & $34.0 \%$ & $31.2 \%$ & 0.65 \\
Sepsis day of life & $26.4 \pm 27.8$ & $38.2 \pm 27.4$ & 0.01 \\
Total antibiotic days & $21.3 \pm 20.3$ & $25.4 \pm 16.3$ & 0.03 \\
highest direct bilirubin & $1.8 \pm 2.6$ & $2.1 \pm 2.9$ & 0.10 \\
Direct bilirubin $\geq 2$ mg/dl & $22.1 \%$ & $28.8 \%$ & 0.13 \\
Peak ALP (IU/dl) & $477.1 \pm 211.6$ & $545.9 \pm 261.5$ & 0.01 \\
UAC days & $7.7 \pm 4.5$ & $7.5 \pm 4.8$ & 0.70 \\
UVC days & $8.9 \pm 4.5$ & $6.6 \pm 4.9$ & $<0.001$ \\
PICC line days & $16.2 \pm 16.9$ & $51.4 \pm 27.8$ & $<0.001$ \\
\hline
\end{tabular}




\begin{tabular}{|c|c|c|c|}
\hline Broviac line days & $9.8 \pm 27.3$ & $2.4 \pm 9.9$ & $<0.001$ \\
\hline Total central line days* & $34.4 \pm 31.3$ & $60.1 \pm 29.5$ & $<0.001$ \\
\hline Chronic lung disease & $67.2 \%$ & $51.6 \%$ & $<0.01$ \\
\hline Mechanical ventilation days & $39.3 \pm 28.5$ & $33.6 \pm 31.1$ & 0.08 \\
\hline Postnatal steroid use & $20.4 \%$ & $16.8 \%$ & 0.42 \\
\hline Hypotension (inotrope use) & $46.6 \%$ & $27.2 \%$ & $<0.001$ \\
\hline Medical PDA & $31.3 \%$ & $44.0 \%$ & 0.01 \\
\hline Surgical PDA (ligation) & $42.5 \%$ & $27.2 \%$ & $<0.01$ \\
\hline Any IVH & $37.0 \%$ & $34.4 \%$ & 0.65 \\
\hline Grade 3/4 IVH & $14.3 \%$ & $12.0 \%$ & 0.64 \\
\hline Any ROP & $82.3 \%$ & $73.6 \%$ & 0.05 \\
\hline ROP laser treatment & $28.9 \%$ & $18.4 \%$ & 0.03 \\
\hline
\end{tabular}

*Excluding UAC days. Abbreviations: CONS (coagulase negative staphylococcal sepsis), ALP (alkaline phosphatase), UAC (umbilical arterial catheter), UVC (umbilical venous catheter), PICC (peripherally inserted central catheter), PDA (patent ductus arteriosus), IVH (intra-ventricular hemorrhage), ROP (retinopathy of prematurity)

In infants who survived to NICU discharge, the SSEF group, had significantly greater body weight ( $2981 \pm 912$ vs. $2694 \pm 842)$ with similar length of NICU stay (110.2 \pm 41 vs. $106.7 \pm 43$ days) (Table 6). Extra-uterine growth restriction [EUGR, $<10^{\text {th }}$ 
percentile of weight for corrected gestational age (CGA)] was significantly less in the SSEF group compared to the controls, while infants with head circumference $<10^{\text {th }}$ percentile at NICU discharge was similar between the groups (Table 6). The SSEF group had significantly lower death ( 4.8 vs. $12.6 \%, p=0.02)$ and combined NEC/death (10.4 vs. $19.4 \%, p=0.03$ ) rates compared to controls (Table 6). However, in subgroup analysis, the significant reduction in combined NEC/death observed in the SSEF group was present only in infants with birth weight $<750 \mathrm{~g}(12.8 \%$ vs. $29.5 \%, \mathrm{p}=0.03)$ while it was similar in the $750-1000$ g group (9.0 vs. $11.5 \%, p=0.66$ ). All the infants in the SSEF group with NEC survived to NICU discharge while $39.4 \%(13 / 33)$ [ $750 \mathrm{~g}=10 / 21(47.6 \%), 750$ $1000 \mathrm{~g}=3 / 12(25 \%)]$ of infants with NEC in the control group died $(p=0.07)$. NEC was an attributable cause of death in $35.1 \%(13 / 37) \quad[<750 \mathrm{~g}=10 / 27(37.0 \%), 750$ $1000 \mathrm{~g}=3 / 10(30 \%)]$ of the controls compared to $0 \%(0 / 6)$ in the SSEF group.

Table 6: Discharge Outcomes of Control and SSEF group ELBW infants

\begin{tabular}{|l|c|c|c|}
\hline & Controls & SSEF group & p value [OR(Cl)] \\
\hline Weight (g)* (median) & $\begin{array}{c}2694.2 \pm 842.5 \\
(2484)\end{array}$ & $\begin{array}{c}2981.2 \pm 912.0 \\
(2885)\end{array}$ & $<0.01$ \\
\hline Weight percentile* & $8.6 \pm 11.9$ & $14.8 \pm 15.1$ & $<0.001$ \\
\hline $\begin{array}{l}\text { Weight <10th } \\
\text { percentile* }\end{array}$ & $75.4 \%$ & $57.1 \%$ & $<0.001$ \\
\hline HC < 10th percentile* & $48.4 \%$ & $38.1 \%$ & 0.10 \\
\hline $\begin{array}{l}\text { Length of stay (days) } \\
\text { (median)* }\end{array}$ & $\begin{array}{c}106.7 \pm 43.5 \\
(99)\end{array}$ & $\begin{array}{c}110.2 \pm 41.5 \\
(104)\end{array}$ \\
\hline Death & $37 / 294(12.6 \%)$ & $6 / 125(4.8 \%)$ & $0.02[0.35(0.11-0.87)]$ \\
\hline
\end{tabular}




\begin{tabular}{|l|c|c|c|}
\hline$<750 \mathrm{~g}$ & $27 / 129(20.9 \%)$ & $5 / 47(10.6 \%)$ & $0.13[0.45(0.16,1.25)]$ \\
$750-1000 \mathrm{~g}$ & $10 / 165(6.0 \%)$ & $1 / 78(1.2 \%)$ & $0.11[0.20(0.03,1.60)]$ \\
\hline Combined NEC/death & $57(19.4 \%)$ & $13(10.4 \%)$ & $0.03[0.48(0.23-0.94)]$ \\
$750 \mathrm{~g}$ & $38 / 129(29.5 \%)$ & $6 / 47(12.8 \%)$ & $0.03[0.35(0.14,0.89)]$ \\
$750-1000 \mathrm{~g}$ & $19 / 165(11.5 \%)$ & $7 / 78(9.0 \%)$ & $0.65[0.76(0.30,1.89)]$ \\
\hline
\end{tabular}

To calculate the propensity scores, the following baseline (birth weight, birth gestation, gender, ethnicity, small for gestational age, mode of delivery, antenatal steroid exposure, pre-eclampsia, maternal diabetes, maternal smoking, substance abuse, magnesium tocolysis, chorioamnionitis, SNAP-PE score, Apgar 1 and 5 minutes, early sepsis, umbilical arterial line days, IVH) and nutritional (human milk as initial milk) variables were included in the multiple logistic regression model. The SSEF group had a mean propensity score of $0.36 \pm 0.12$, whereas the control group had a mean propensity score of $0.27 \pm 0.15(p<0.001)$. In both the PS- matched groups $(1: 1$ match of all SSEF group infants to the nearest PS matched controls without replacement) and the PSweighted groups, all the covariates included in the model were well balanced (none with $\mathrm{p}$ value $<0.05)$. The important PS-adjusted outcomes using various PS methods are summarized in Table 7. 
Table 7: Propensity score adjusted outcomes in SSEF group compared to controls

\begin{tabular}{|c|c|c|c|c|}
\hline Outcome & Unadjusted & $\begin{array}{c}\text { 1:1 PS } \\
\text { matching }\end{array}$ & PS weighting & $\begin{array}{l}\text { Direct PS } \\
\text { adjustment }\end{array}$ \\
\hline NEC & $\begin{array}{c}0.47 \\
(0.17-1.12)\end{array}$ & $\begin{array}{c}0.44 \\
(0.17-1.11)\end{array}$ & $\begin{array}{c}0.45 \\
(0.19-1.10)\end{array}$ & $\begin{array}{c}0.41 \\
(0.15,1.01)\end{array}$ \\
\hline Death & $\begin{array}{c}0.35 \\
(0.11-0.87)\end{array}$ & $\begin{array}{c}0.30 \\
(0.11-0.78)\end{array}$ & $\begin{array}{c}0.27 \\
(0.11-0.68)\end{array}$ & $\begin{array}{c}0.28 \\
(0.10,0.66)\end{array}$ \\
\hline $\begin{array}{l}\text { Combined } \\
\text { NEC/death }\end{array}$ & $\begin{array}{c}0.48 \\
(0.23-0.94)\end{array}$ & $\begin{array}{c}0.42 \\
(0.21-0.86)\end{array}$ & $\begin{array}{c}0.42 \\
(0.22-0.83)\end{array}$ & $\begin{array}{c}0.40 \\
(0.19,0.80)\end{array}$ \\
\hline $\begin{array}{l}\text { Late-onset } \\
\text { Sepsis }\end{array}$ & $\begin{array}{c}0.92 \\
(0.60,1.41)\end{array}$ & $\begin{array}{c}0.85 \\
(0.51,1.40)\end{array}$ & $\begin{array}{c}0.87 \\
(0.55,1.36)\end{array}$ & $\begin{array}{c}0.84 \\
(0.50,1.39)\end{array}$ \\
\hline Cholestasis & $\begin{array}{c}1.47 \\
(0.92,2.38)\end{array}$ & $\begin{array}{c}1.12 \\
(0.64,1.95)\end{array}$ & $\begin{array}{c}1.27 \\
(0.76,2.13)\end{array}$ & $\begin{array}{c}1.08 \\
(0.62,1.90)\end{array}$ \\
\hline $\begin{array}{l}\text { Peak ALP } \\
\text { (IU/dl) }\end{array}$ & $\begin{array}{c}68.8 \\
(20.9,116.9)\end{array}$ & $\begin{array}{c}64.0 \\
(3.6,124.3)\end{array}$ & $\begin{array}{c}68.1 \\
(12.6,123.6)\end{array}$ & $\begin{array}{c}61.2 \\
(0.62,121.8)\end{array}$ \\
\hline $\begin{array}{l}\text { Discharge } \\
\text { weight(g)* }\end{array}$ & $\begin{array}{c}287.1 \\
(98.6,475.6)\end{array}$ & $\begin{array}{c}132.0 \\
(-144.6\end{array}$ & $\begin{array}{c}202.0 \\
(-18.0,422.0)\end{array}$ & $\begin{array}{c}115.3 \\
(-133.8,364.4)\end{array}$ \\
\hline $\begin{array}{l}\text { Length of stay } \\
\text { (days)* }\end{array}$ & $\begin{array}{c}3.50 \\
(-5.9,12.8)\end{array}$ & $\begin{array}{c}6.12 \\
(-4.1,16.4)\end{array}$ & $\begin{array}{c}0.89 \\
(-9.0,10.7)\end{array}$ & $\begin{array}{c}5.7 \\
(-4.8,16.1)\end{array}$ \\
\hline
\end{tabular}


*Only infants surviving to NICU discharge included " direct bilirubin $\geq 2 \mathrm{mg} / \mathrm{dl}$

Abbreviations: PS (propensity score), NEC (necrotizing enterocolitis), ALP (alkaline phosphatase). Odds ratio with confidence interval for proportions (NEC, death, combined NEC/death, late-onset sepsis and cholestasis), estimated mean difference with confidence interval for continuous variables (peak ALP, discharge weight and length of stay)

The incidence of NEC was not significantly different between the SSEF group and controls in PS score adjusted analysis. The PS-adjusted all-cause death and combined NEC/death were significantly lower in the SSEF group compared to controls. In subgroup analysis of infants with birth weight $<750 \mathrm{~g}$, the SSEF group had a significantly lower incidence of NEC [OR $0.14(0.007,0.74)]$ and combined NEC/death [OR 0.32(0.11, 0.82)] compared to controls, while both of these outcomes were similar in the $750-1000 \mathrm{~g}$ weight group. The PS-adjusted incidence of late-onset sepsis and cholestasis were similar between the groups, however peak ALP level was significantly higher in the SSEF group compared to the controls. In infants who survived to NICU discharge, the PSadjusted discharge weight and length of stay were similar between the SSEF group and controls.

Since there were significant differences in co-morbidities between the SSEF and control groups (Table 5) that may be potentially associated with death, an additional logistic regression was performed to determine the adjusted SSEF group effect on the combined NEC/death outcome using all the covariates potentially related to combined 
NEC/death with a $p$ value $\leq 0.10$ in the unadjusted analysis (total antibiotics days, cholestasis, peak ALP, total central line days, chronic lung disease, inotropic use, PDA ligation, ROP laser). When controlling for these factors, being in the SSEF group was independently associated with significant reduction in combined NEC/death [OR 0.30 $(\mathrm{Cl}: 0.12,0.71), \mathrm{p}<0.01]$

\section{DISCUSSION}

Our study demonstrates that a standardized slow enteral feeding protocol with maternal human milk/preterm formula reduces the incidence of NEC and combined NEC/death in infants with birth weight $<750 \mathrm{~g}$. These results are comparable to studies that used an exclusive human milk diet for reducing NEC. However, due to the prolonged nature of the SSEF protocol, these infants were exposed to longer central line and PN days. Despite taking longer to achieve full enteral feeding with the SSEF protocol, these infants demonstrated comparable weight gain at NICU discharge without prolonging their hospital stay.

Epidemiological studies have found that compared to non-Hispanic white infants, non- Hispanic African American infants have a higher incidence of NEC. ${ }^{21}$ The incidence of NEC is also reported to be higher in male infants. ${ }^{22}$ Holman et al described the trends and risk factors for infant mortality in the USA and showed that death from NEC was highest in VLBW infants who were black and male. ${ }^{23}$ In addition, the SNAP-PE score in the range of 40-49 observed in our study population represents a high neonatal severity illness score with a predicted mortality rate of $15.9 \%{ }^{20}$ Thus, the demographic 
characteristics of ELBW infants in our study (higher proportion of African American male infants) represent a group at particularly high risk for developing NEC.

Observational data suggests that delaying the introduction of enteral feeds until 510 days postnatally reduces the risk of NEC in VLBW infants. ${ }^{12,13,15}$ However, prolonged NPO status may cause atrophy of the intestinal mucosa, delayed development of absorptive function, decreased motility and development of pro-inflammatory changes. ${ }^{24}$ In various observational studies, infants who received early trophic feeds were reported to have better feeding tolerance, improved growth, reduced length of hospitalization and decreased likelihood of sepsis compared to infants who received delayed enteral feeds. ${ }^{25,26,27}$ However, these potential short-term benefits of early enteral feeding were not shown to demonstrate a difference in preventing NEC. ${ }^{16}$ A meta-analysis of 9 RCTs did not find a significant difference in NEC rate, time to regain birth weight, time to reach full enteral feeds, incidence of invasive infection, duration of hospital stay or all-cause mortality between early trophic feeding and enteral fasting (defined as NPO for 5-7 days). ${ }^{16}$ The largest RCT that included ELBW infants randomized appropriately grown 26-30 weeks gestation infants into enteral fasting or trophic feeding for the first 14 days of life. ${ }^{28}$ There was no difference in NEC rate $(15.9 \%$ vs. 11.2\%) and days to reach full enteral feeds (35 vs. 32) between trophic feeding and enteral fasting groups. ${ }^{28}$ However, the primary outcome of the several included RCTs was not the incidence of NEC, but rather feeding intolerance or time to reach full enteral feeds. Additionally, only a minority of the participants in the included trials were ELBW infants. In our study, the average initial NPO period was only 3 days longer in the 
SSEF group compared to controls (13 vs. 10) and the days to reach full enteral feeds was significantly longer ( $63.6 \pm 19.1$ vs. $35.1 \pm 19.3$ )(Table 3 ). This suggests that slow feeding advancement is the more likely associated with NEC reduction rather than the initial NPO period.

An immature enteric nervous system and intestinal dysmotility warrants gradual and cautious increments in enteral feedings. Aggressive enteral feeding advancement is a risk factor associated with NEC. ${ }^{29,30}$ However, a recent meta-analysis of 5 RCTs between slow advancement $(<15-20 \mathrm{ml} / \mathrm{kg} /$ day) versus faster advancement (30-35 $\mathrm{ml} / \mathrm{kg} /$ day) did not detect any significant difference in NEC or all-cause mortality. ${ }^{16}$ Infants who had slower advancement took longer to regain birth weight (difference of 26 days) and to reach full enteral feeds (difference of 2-5 days). ${ }^{16}$ However, ELBW infants were only included in two studies and both had broad exclusion criteria. ${ }^{17,18}$ One RCT that considered NEC as the primary outcome evaluated the effect of stable (20 $\mathrm{ml} / \mathrm{kg} /$ day without advancement) versus advancing $(20 \mathrm{ml} / \mathrm{kg} /$ day to goal 140 $\mathrm{ml} / \mathrm{kg} /$ day) feeding volumes for a 10 -day period in non-SGA VLBW infants. ${ }^{30}$ Enteral feeds were initiated at an average age of 10 days as feeds were initiated only after removal of umbilical catheters and discontinuation of inotropic medications. Only onethird of the infants received human milk and the remainder were fed $24 \mathrm{cal} / \mathrm{oz}$ preterm formula. This study was prematurely terminated as they found a significantly higher incidence of NEC in infants fed advancing volumes compared with those fed with a minimal enteral feeding volume (10.4 vs. $1.4 \%, 7 p=0.03) .{ }^{30}$ Since it is difficult to blind the caregivers to the group assignment, measurement bias may have overestimated the 
NEC rate in the advancing feed group. However, the large difference in NEC rate suggests that one should be cautious with aggressive feeding advancement in the early days of enteral feeding especially when using formula.

In a recently reported multicenter RCT, the incidence of NEC and surgical NEC in preterm infants with birth weight $<1250 \mathrm{~g}$, exclusively fed human milk feeding was $5.8 \%$ and $1.4 \%$, compared to $15.9 \%$ and $10.1 \%$ in infants who were fed with human milk supplemented with bovine milk-based products (Sullivan, 2010). ${ }^{31}$ The infants included in this study were less at risk for NEC (21.6\% African-American infants, 9.6\% SGA infants, average birth weight 925 g) compared to our study (57.6\% African-American infants, $25.6 \%$ SGA infants, average birth weight $766 \mathrm{~g})$. The rates of feeding in the Sullivan study [up to 5 days of trophic feeding $(10-20 \mathrm{ml} / \mathrm{kg} / \mathrm{day})$ followed by $10-20 \mathrm{ml} / \mathrm{kg} /$ day of feed advancement] was also 'slow' as per the definition set by Cochrane reviews $(<24$ $\mathrm{ml} / \mathrm{kg} /$ day feed advancement). ${ }^{31}$ The days to full enteral feeding and days of PN (22 and 21) were significantly less compared to our study (63 and 62). Subgroup data $(<1000 \mathrm{~g}$ birth weight) from this study was not available to compare other outcomes such as like sepsis, cholestasis, central line days and hospital length of stay. With our SSEF protocol, we have achieved a similar rate of NEC to infants fed on an exclusively human milkbased diet. In Sullivan's study, even after following a standardized feeding regimen, the high rate of NEC (15.9\%) in infants who received human milk supplemented with bovine milk-based products suggests that a much slower advancement of feeds such as in our SSEF protocol may be necessary to reduce NEC in infants receiving bovine milk- based formula or fortifiers. 
Though no RCT has compared the effect of mother's own milk (MM) versus formula on NEC or death, there is widespread consensus about the short- and long-term benefits of $\mathrm{MM}$ in preterm infants including its protective effect on NEC. ${ }^{32,33} \mathrm{~A}$ recently reported quality improvement project in California to increase human milk use in VLBW infants resulted in a reduction of NEC from $7 \%$ to $2.4 \% .{ }^{34}$ In another prospective cohort study of VLBW infants, infants who received $\geq 50 \%$ enteral feeds as human milk within the first 14 days of life had one-sixth the odds of developing NEC compared to infants who received less than $50 \%$ enteral feeds with human milk (3.2 vs. $10.6 \%, \mathrm{OR}=0.17, \mathrm{Cl}$ : 0.04 to $0.68, p=0.01) .^{35}$ The higher human milk initiation rate in the SSEF group compared to controls could be a potential confounder in our study, but this difference was balanced by PS methods and the outcomes were unchanged after PS analysis. If MM is not available, the AAP policy on breast feeding advocates donor breast milk (DBM) as a suitable alternative feeding. ${ }^{33}$ A meta-analysis of studies comparing DBM and formula demonstrated that preterm infants fed with formula had more than twice the odds of NEC compared to infants fed with DBM. ${ }^{36,37}$ However, these studies included only a minority of extremely preterm infants and the effects of DBM combined with HMF were not evaluated. ${ }^{31} \mathrm{~A} R C T$ that compared fortified pasteurized DBM and preterm formula, both used as supplements when MM was not available, did not find a protective effect of DBM on the combined incidence of late-onset sepsis and NEC. ${ }^{32}$ Similarly in Sullivan's study, a high incidence of NEC (15.6\%) was observed in infants who received human milk (MM or DBM) supplemented with bovine milk-based HMF. ${ }^{31}$ The use of MM/DBM may help in the earlier initiation and attainment of full enteral feeding, 
but slowing the feeding advancement after supplementing with bovine milk HMF may help the immature intestine to adapt and reduce the risk for developing NEC. Dietdependent modification to the standardized feeding regimen may be needed for sustained prevention of NEC, e.g. - slower advancement of feeds in infants fed with bovine milk-based products alone or mixed with MM/DBM compared to an exclusive human milk diet.

In a population-based cohort study of ELBW infants, NEC occurred at an average postnatal age of 32 days $^{38}$, similar to our controls. The nearly doubled time to the onset of NEC in the SSEF group suggests that delaying the establishment of enteral feeds also delays the onset of NEC. This is also consistent with the observation that NEC commonly occurs when feeding volume exceeds $100 \mathrm{ml} / \mathrm{kg} / \mathrm{day} .{ }^{17,30,39}$ The need for surgical intervention is often used as a surrogate marker for the severity of NEC. ${ }^{6,7,40}$ About $50 \%$ of infants $<750 \mathrm{~g}$ in our controls developed surgical NEC compared to none in the SSEF group (Table 4). It is plausible that by using a SSEF protocol these extremely premature infants are more physiologically mature and have better protective mechanisms to tolerate the delayed onset of NEC leading to a lower risk of progressing to advanced NEC requiring surgical intervention.

Since conservative feeding strategies are associated with prolonged use of PN and central line days, they may alter other competing outcomes, especially the rate of nosocomial infections. There was no increase in the incidence of late-onset sepsis in the SSEF group compared to controls; however, the adoption of bundle strategies to 
prevent central line-associated bloodstream infections (CLABSI) potentially helped us to control the infection rate during the prospective study period. The statistically significantly higher peak serum alkaline phosphatase levels in the SSEF group (545 vs. $477 \mathrm{IU} / \mathrm{dl}$ in controls) reflect the potential nutrition-related harm associated with the SSEF protocol, however, the magnitude of difference may not be clinically meaningful. We believe that the potential benefits of the SSEF protocol outweigh the risks as these infants had significant reduction in NEC/death and demonstrated comparable weight gain and head growth at NICU discharge without prolonging the NICU stay.

In the Sullivan study of infants' $\leq 1250$ g, the combined outcome of NEC/death was significantly less common in exclusively human milk fed infants compared to human milk supplemented with bovine milk-based product (7.3 vs. $20 \%) .{ }^{31}$ With the SSEF protocol, we have demonstrated a similar reduction in NEC/death in ELBW infants (10.4 vs. $19.4 \%)$. This is even more significant as most of the reduction in NEC/death happened in infants with birth weight $<750 \mathrm{~g}(12.8$ vs. $29.5 \%)$ - the group with a reported NEC mortality rate of $40-60 \%$ as per the largest NEC study cohort published. ${ }^{6}$ Based on our study results, the number of infants with birth weight $<750 \mathrm{~g}$ that would be needed to treat with the SSEF protocol to prevent one case of NEC is 7 and the number needed to treat to prevent one combined NEC/death is 6 .

One of the strengths of our study is the use of NEC incidence as the primary outcome. Most of the previous single center studies were underpowered to detect a difference in NEC rate due to the relatively low incidence of NEC. However, our study is not without limitations. Only about one-third of the infants remained on human milk at 
full enteral feeds in spite of higher human milk initiation rate $(80.2 \%)$. Our results are not isolated as one study reported that only $30 \%$ of mothers were able to supply $100 \%$ of their extremely premature infants' needs. ${ }^{32}$ This suggests that our outcomes are not generalizable to population fed mainly with human milk diet. Since our study spans more than a decade, it is possible that practice changes in the care of ELBW infants have affected the outcomes reported. For example, more medical treatment of PDA compared to primary PDA ligation and earlier extubation and aggressive non-invasive ventilation in the SSEF group reflects current neonatal practice. Also, we have reported only the short-term outcomes associated with the SSEF protocol as the study was not designed to assess the long-term neuro-developmental outcomes.

\section{CONCLUSION}

NEC is a devastating disease with high morbidity and mortality. Enteral feeding practices and the type of milk used represent two major modifiable risk factors of NEC in ELBW infants. Our study demonstrates that when using human milk or preterm formula, following a standardized slow enteral feeding protocol helps to reduce NEC and combined NEC/death in infants with birth weight $<750 \mathrm{~g}$. We believe the benefits of reducing the combined $\mathrm{NEC} /$ death rate outweighs the potential nutrition-related harm associated with delayed initiation and slower advancement of enteral feeds especially when using formula as initiation milk. However, because the etiology of NEC is multifactorial, only adequately powered, vigorously conducted randomized trials with 
sufficient follow-up can conclusively assess the effect of slow feeding advancement on NEC and other related short- and long-term outcomes in ELBW infants. 


\section{BIBLIOGRAPHY}

${ }^{1}$ Neu J, Walker WA. Necrotizing enterocolitis. N Engl J Med. 2011;364(3):255-264.

2 Athalye-Jape G, More K, Patole S. Progress in the field of necrotising enterocolitis-year 2012. J Matern Fetal Neonatal Med. 2013;26(7):625-632

3 Holman RC, Stoll BJ, Curns AT, Yorita KL, Steiner CA, Schonberger LB. Necrotising enterocolitis hospitalisations among neonates in the United States. Paediatr Perinat Epidemiol. 2006;20(6):498-506

${ }^{4}$ Fitzgibbons SC, Ching Y, Yu D, et al. Mortality of necrotizing enterocolitis expressed by birth weight categories. J Pediatr Surg. 2009;44(6):1072-1075; discussion 10751076

5 Clark RH, Gordon P, Walker WM, et al. Characteristics of patients who die of necrotizing enterocolitis. J Perinatol.2012; 32(3):199-204.8.

6 Hull MA, Fisher JG, Gutierrez IM, Horbar JD, Jaksic T, et al. Mortality and management of surgical necrotizing enterocolitis in very low birth weight neonates: a prospective cohort study. J Am Coll Surg. 2014 Jun;218(6):1148-55.

${ }^{7}$ Lin PW, Nasr TR, Stoll BJ. Necrotizing enterocolitis: recent scientific advances in pathophysiology and prevention. Seminars in Perinatology, 32(2): 70-82, 2008

8 Stoll BJ, Hansen NI, Bell EF, Shankaran S, et al. Neonatal outcomes of extremely preterm infants from the NICHD Neonatal Research Network. Pediatrics (2010) 126:443-456..

9 Bisquera JA, Cooper TR, Berseth CL Impact of necrotizing enterocolitis on length of stay and hospital charges in very low birth weight infants. Pediatrics. 2002; 109(3):423-428.).

10 Henry MC, Moss RL. Necrotizing enterocolitis. Annual Review of Medicine, 60: 111-24, 2009

11 Neu J, Mihatsch W. Recent developments in necrotizing enterocolitis. JPEN J Parenter Enteral Nutr. 2012;36(1 Suppl):30S-35S.

12 Henderson G, Craig S, Brocklehurst P, McGuire W. Enteral feeding regimens and necrotising enterocolitis in preterm infants: a multicentre case-control study. Arch Dis Child Fetal Neonatal Ed. 2009;94(2):F120-123 
13 Patole SK, de Klerk N. Impact of standardised feeding regimens on incidence of neonatal necrotising enterocolitis: a systematic review and meta-analysis of observational studies. Arch Dis Child Fetal Neonatal Ed 2005; 90:F147-51

${ }^{14}$ Fanaroff AA, Stoll BJ, Wright LL, et al. Trends in neonatal morbidity and mortality for very low birth weight infants. Am J Obstet Gynecol 2007; 196:147.e1-8.

15 Pietz J, Achanti B, Lilien L et al. Prevention of Necrotizing Enterocolitis in Preterm Infants: A 20-Year Experience. Pediatrics; 119(0), e164-e170

16 Morgan J, Young L, McGuire W. Slowly advancing milk feeds does not reduce the risk of necrotising enterocolitis in very low birth weight infants. Cochrane review, March 2013

17 Rayyis SF, Ambalavanan N, Wright L, Carlo WA.Randomized trial of "slow" versus "fast" feed advancements on the incidence of necrotizing enterocolitis in very lowbirth weight infants. J Pediatr. 1999 Mar;134(3):293-7.

18 Karagol BS, Zenciroglu A, Okumus N, Polin RA.Randomised controlled trial of slow versus rapid enteral feeding advancements on the clinical outcomes of preterm infants with 750-1250g. JPEN J Parenter Enteral Nutr. 2013; 37(2):223-8.

19 Walsh MC, Kliegman RM. Necrotizing enterocolitis: treatment based on staging criteria. Pediatr Clin North Am. 1986 Feb;33(1):179-201.

20 Richardson DK, Corcoran JD, Escobar GJ, Lee SK. SNAP-II and SNAPPE-II: Simplified newborn illness severity and mortality risk scores. J Pediatr. 2001; 138(1):92-100.

${ }^{21}$ Llanos AR, Moss ME, Pinzon MC, Dye T, Sinkin RA, Kendig JW. Epidemiology of neonatal necrotizing enterocolitis: a population-based study. Paediatr Perinat Epidemiol. 2002; 16:342-349

22 Guner YS, Friedlich P, Wee CP, Dorey F, Camerini V, Upperman JS. State-based analysis of necrotizing enterocolitis outcomes. J Surg Res. 2009;157(1):21-29

23 Holman RC, Stoll BJ, Clarke MJ, Glass RI. The epidemiology of necrotizing enterocolitis infant mortality in the United States.Am J Public Health 1997; 87: 2026-31.

${ }^{24}$ Kudsk KA. Current aspects of mucosal immunology and its influence by nutrition. Am J Surg. 2002; 183:390-398.

${ }^{25}$ De Silva A, Jones PW, Spencer SA (2004). Does human milk reduce infection rates in preterm infants? A systematic review. Arch Dis Child Fetal Neonatal Ed 89, F509F513 
${ }^{26}$ Flidel-Rimon $\mathrm{O}^{1}$, Branski D, Shinwell ES. The fear of necrotizing enterocolitis versus achieving optimal growth in preterm infants--an opinion. Acta Paediatr. 2006 Nov; 95(11):1341-4.

${ }^{27}$ McClure RJ. Trophic feeding of the preterm infant. Acta Paediatr Suppl. 2001 Mar; 90(436):19-21.

28 Schanler RJ, Shulman RJ, Lau C. Feeding strategies for premature infants: Beneficial outcomes of feeding fortified human milk vs. preterm formula. Pediatrics 1999;103:1150-7

${ }^{29}$ Anderson DM, Kliegman RM. The relationship of neonatal alimentation practices to the occurrence of endemic necrotizing enterocolitis. Am J Perinatol. 1991; 8:6267.35 .

${ }^{30}$ Berseth CL, Bisquera JA, Paje VU. Prolonging small feeding volumes early in life decreases the incidence of necrotizing enterocolitis in very low birth weight infants. Pediatrics. 2003; 111:529-534.

${ }^{31}$ Sullivan S, Schanler RJ, Kim JH, Patel AL, et al. An exclusively human milk-based diet is associated with a lower rate of necrotizing enterocolitis than a diet of human milk and bovine milk-based products. J Pediatr. 2010 Apr; 156(4):562-7

${ }^{32}$ Schanler RJ, Lau C, Hurst NM, Smith EO. Randomized trial of donor human milk versus preterm formula as substitutes for mothers' own milk in the feeding of extremely premature infants. Pediatrics. 2005;116(2):400-406

33 Eidelman AI, Schanler RJ.Section on Breastfeeding Executive Committee. Breastfeeding and the use of human milk: policy statement. Pediatrics 2012;129:e827-41

${ }^{34}$ Lee HC, Kurtin PS, Wight NE, et al. A quality improvement project to increase breast milk use in very low birth weight infants. Pediatrics 2012; 130:e1679-87.)

35 Sisk PM, Lovelady CA, Dillard RG, Gruber KJ, O'Shea TM. Early human milk feedings associated with a lower risk of necrotizing enterocolitis in very low birth weight infants. J Perinatol. 2007;27(7):428-433

${ }^{36}$ Quigley M, McGuire W. Formula versus donor breast milk for feeding preterm or low birth weight infants. Cochrane Database Syst Rev. 2014;4:CD002971

${ }^{37}$ Boyd CA, Quigley MA, Brocklehurst P. Donor breast milk versus infant formula for preterm infants: a systematic review and meta-analysis. Arch Dis Child Fetal Neonatal Ed 2007;92:F169-75

${ }^{38}$ Yee WH, Soraisham AS, Shah VS, et al. Incidence and timing of presentation of necrotizing enterocolitis in preterm infants. Pediatrics. 2012;129(2):e298-304. 
${ }^{39}$ Owens L, Berseth. Is there a volume threshold for enteral feeding? Pediatric rese 1996.

40 Guthrie SO, Gordon PV, Thomas V, Thorp JA, Peabody J, Clark RH. Necrotizing enterocolitis among neonates in the United States. J Perinatol 2003; 23: 278-85. 